\title{
DUAL MASS FLYWHEEL PARAMETER IDENTIFICATION
}

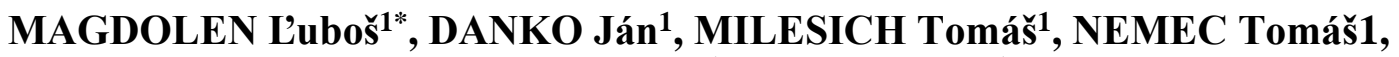 \\ SLOBODA Karol ${ }^{1}$, BUCHA Jozef ${ }^{1}$
}

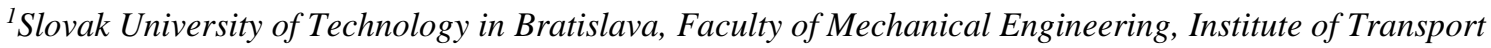 \\ Technology and Engineering Design, Nám. slobody 17,812 31 Bratislava, Slovakia, \\ e-mail:lubos.magdolen@stuba.sk
}

\begin{abstract}
Reducing emissions brings changes in the design of internal combustion engines and thus new challenges for dual-mass flywheels (DMF) in terms of Noise Vibration and Harshness (NVH). The first part of the article describes a simple model of a centrifugal pendulum. Consequently, a more complicated DMF dynamic model involves friction between the spring components. The second part of the article deal with the multibody model of DMF using a CAD model. The dynamic model consists of a torsion spring and two bodies. The model is compared with the experimental method, which is also described in the paper.
\end{abstract}

KEYWORDS: Dual mass flywheel, Pendulum, Multibody model, Noise Vibration and Harshness

\section{Introduction}

Manufacturers in the automotive industry are increasingly confronted with reducing the weight of components in order to achieve the often-mentioned targets of fuel consumption and thus reducing $\mathrm{CO} 2$ emissions. The result is the production of smaller engines with fewer cylinders, which reduces the power output by increasing the pressures in the cylinders and thus more efficient combustion. As a result, stronger torsional vibrations occur on the crankshaft, which is further transmitted to the drive train [1].

In the current state of torsional vibration damping, the market offers many processes from various materials from single mass flywheel to more complex systems such as fluid torsional vibration damper or overrunning damper isolator pulleys. However, the most widely used damper in the automotive industry is still the Dual-mass flywheel.

The first part of the article will discuss the simple dynamic system of pendulum vibration absorber to understand the use of a dual-mass flywheel. In the second part, the physical behaviour with friction of DMF (dual mass flywheel) is evident. The third part contains a basic explanation of DMF as a multibody model, whose simulation is compared with the measured values. The fourth and fifth chapter concerns the measuring device and subsequent processing, which can induce a simulated excitation of the motor torque and determine the effect of the motor on the damping system and thus eliminate a deeper analysis of $\mathrm{NVH}$ issues.

\section{Equations of motions of centrifugal pendulum vibration absorber}

The initial designs of the simulation model are realized using the equations centrifugal pendulum vibration absorber - simple type, which is predicted by Figure 5. The equations of motion are based on the Lagrange formulation from the general coordinates:

$$
\begin{aligned}
& x_{1}=x_{1}\left(q_{1}, q_{2}, t\right) \\
& x_{2}=x_{2}\left(q_{1}, q_{2}, t\right)
\end{aligned}
$$

$q_{1,}=\varphi-$ rotation angle 
$q_{2,}=\xi$-angle of the pendulum relative to the point of attachment of the pendulum to the rotor

The overall derivation of the equations of motion is not included here. Lagrange's equations of motion, when we do not consider the dissipative (Rayleigh) function, can be expressed in the form:

$$
\begin{gathered}
\frac{d}{d t}\left(\frac{\partial E_{k}}{\partial \dot{q}_{j}}\right)-\frac{\partial E_{k}}{\partial q_{j}}+\frac{\partial E_{p}}{\partial q_{j}}=0 \\
\frac{d}{d t}\left(\frac{\partial L}{\partial \dot{q}_{j}}\right)-\frac{\partial L}{\partial q_{j}}=0 \\
L=E_{k}-E_{p}
\end{gathered}
$$

These equations are called second-order Lagrange equations of motion, represent a system of second-order linear differential equations. In the CPVA system, there are:

$$
\begin{gathered}
\frac{d}{d t}\left(\frac{\partial L}{\partial \dot{\varphi}}\right)-\frac{\partial L}{\partial \varphi}=0 \\
\frac{d}{d t}\left(\frac{\partial L}{\partial \dot{\xi}}\right)-\frac{\partial L}{\partial \xi}=0
\end{gathered}
$$

Equations (6) and (7) are further used to describe the dynamic properties of CPVAs. Because the equations are second order, it is not possible to insert them directly into the solution, it was necessary to introduce new variables and formulate them as equations of motion of the first order ODR system [2].

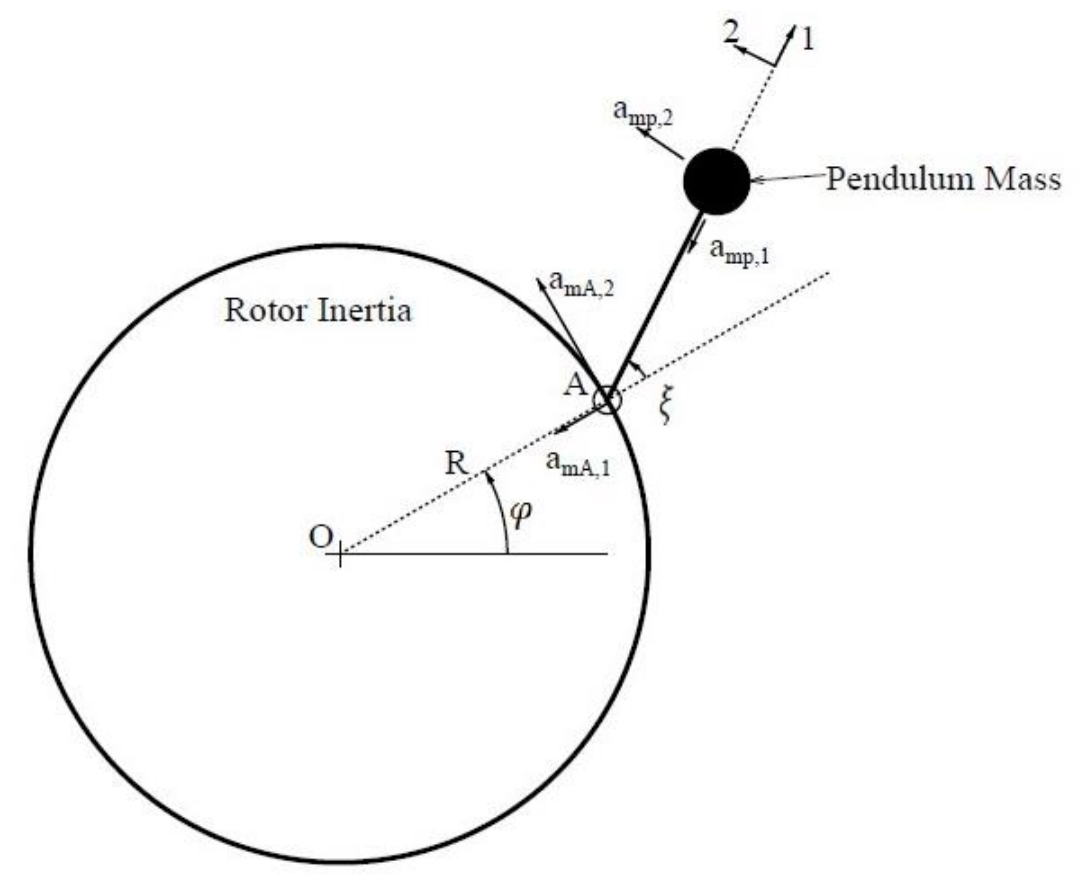

Fig. 1 Simple model of pendulum with point mass

Then the Lagrange equations have the form:

$$
\begin{aligned}
& k_{1}(\xi) \ddot{\varphi}+k_{2}(\xi) \ddot{\xi}+k_{3}(\xi) \dot{\varphi}^{2}+k_{4}(\xi) \dot{\xi}^{2}+k_{5}(\xi) \dot{\varphi} \dot{\xi}=0 \\
& k_{6}(\xi) \ddot{\varphi}+k_{7}(\xi) \ddot{\xi}+k_{8}(\xi) \dot{\varphi}^{2}+k_{9}(\xi) \dot{\xi}^{2}+k_{10}(\xi) \dot{\varphi} \dot{\xi}=0
\end{aligned}
$$


We have introduced a new variable to reduce the system of equations to the first - order:

$$
\begin{gathered}
y(t)=\left[\begin{array}{l}
\varphi(t) \\
\dot{\varphi}(t) \\
\xi(t) \\
\dot{\xi}(t)
\end{array}\right] \\
\frac{d}{d t}(y(t))=\dot{y}(t)=\left[\begin{array}{l}
\varphi(t) \\
\dot{\varphi}(t) \\
\xi(t) \\
\dot{\xi}(t)
\end{array}\right] \\
A \dot{y}(t)=f
\end{gathered}
$$

Then, with equations (8) and (9), matrices written in (10) are formed:

$$
f=\left[\begin{array}{c}
\dot{y}(t)=\left[\begin{array}{c}
\dot{\varphi}(t) \\
\ddot{\varphi}(t) \\
\dot{\xi}(t) \\
\ddot{\xi}(t)
\end{array}\right] \\
y(2) \\
-\left(k_{3}(y(3)) y(2)^{2}+k_{4}(y(3)) y(4)^{2}+k_{5}(y(3)) y(2) y(4)\right) \\
y(2) \\
-\left(k_{3}(y(3)) y(2)^{2}+k_{4}(y(3)) y(4)^{2}+k_{5}(y(3)) y(2) y(4)\right)
\end{array}\right]
$$

The formulation is transposed into the system of first-order differential equations.

$$
\dot{y}(t)=A^{-1} f
$$

\section{Mathematical model of dynamic properties of DMF}

The primary flywheel is defined from the first law of the moment of momentum, where the clustered mass with the moment of inertia had the designation primary, i.e. the moment of inertia $I_{p r i}$ and the angular acceleration $\varphi_{\text {pri }}$. An analogous similar designation will apply to the second part of the flywheel, $I_{s e c}$ and $\varphi_{\text {sec }}$. The relative acceleration of the secondary to the primary represents $\ddot{\varphi}_{D M F}[3]$.

The primary flywheel equations of the first law of conservation of angular momentum:

$$
I_{\text {pri }} \varphi_{\text {pri }}=M_{E}-\sigma_{1}^{P} M_{S, 1}+\sigma_{6}^{P} M_{S, 6}+M_{\text {fric }, \text { pri }}
$$

Secondary flywheel equation of the first law of conservation of angular momentum:

$$
I_{\text {pri }}\left(\varphi_{\text {pri }}+\varphi_{\text {sec }}\right)=-M_{C}-\sigma_{1}^{S} M_{S, 1}+\sigma_{6}^{S} M_{S, 6}+M_{\text {fric }, \text { sec }}
$$




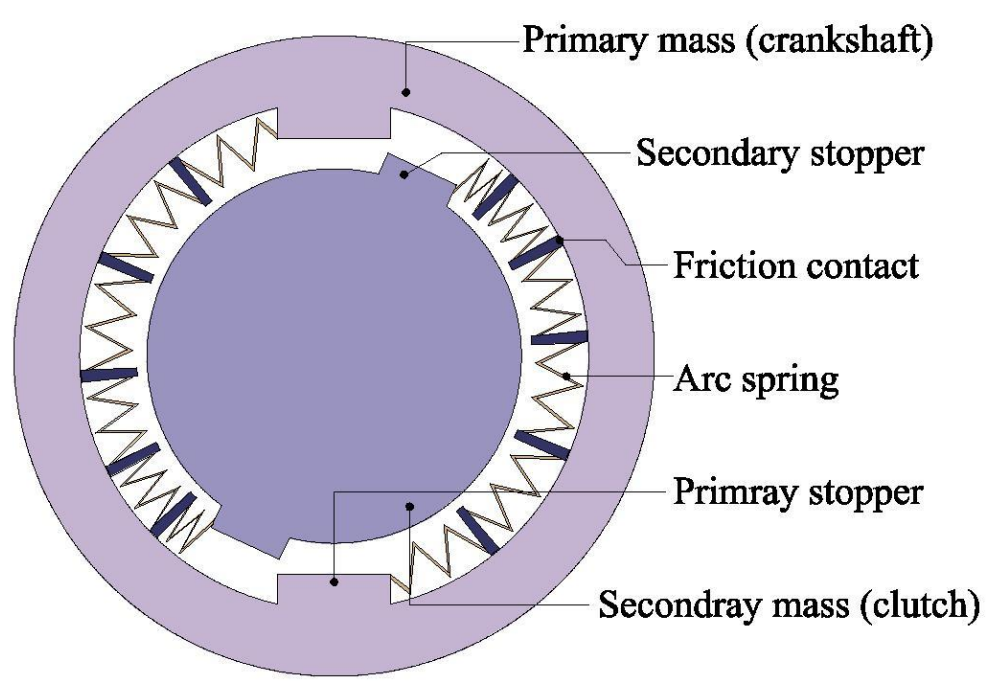

Fig. 2 Basic naming of elements of compressed DMF

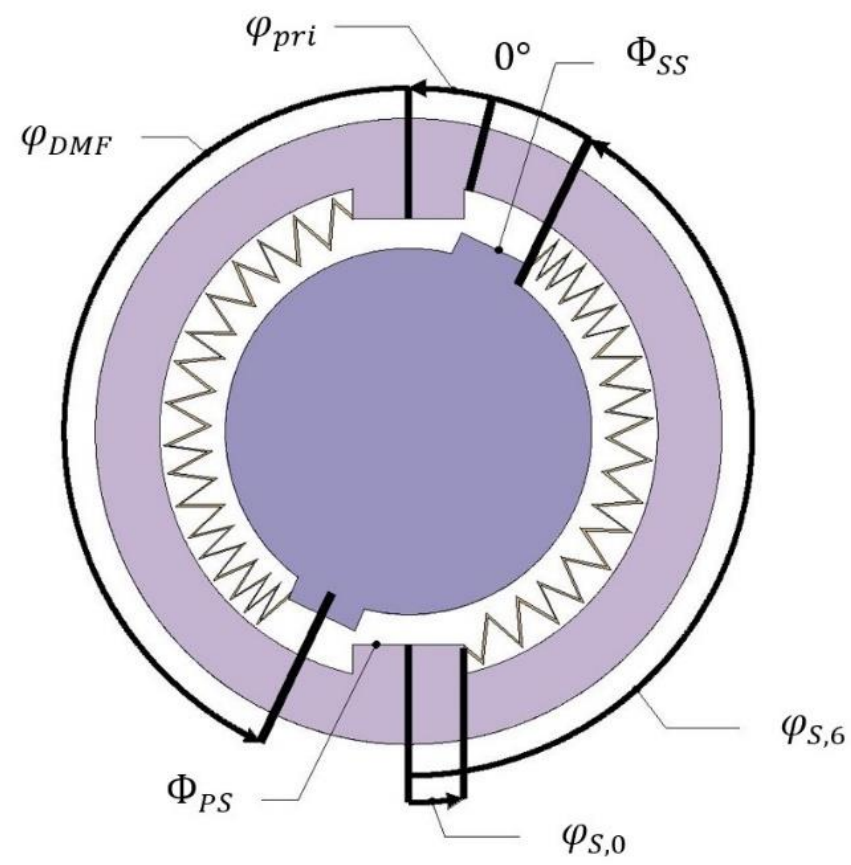

Fig. 3 The coordinate system for flywheel and spring model $\varphi^{*}$ denotes constants while $\Phi^{*}$ are variable angles.

In the equation, $M_{E}$ denotes the engine torque and $M_{C}$ indicates the clutch torque. The moments of springs $M_{S, 1}, M_{S, 6}$ are defined below. Those moments influence angular momentum to the flywheels according to the switching functions $\sigma_{1}^{P}, \sigma_{6}^{P}, \sigma_{1}^{S}$ and $\sigma_{6}^{S}$. These functions $\sigma_{*}^{*}$ $\in\{0,1\}$ are $\sigma_{*}^{*}=1$, when the spring touches one of the stoppers of the flywheel as a normal case. If the spring touches the primary mass stopper, then $\sigma_{1}^{P}=1$. If the spring's back end touches the other primary, then $\sigma_{6}^{P}=1$. The same is true for the switching functions of the secondary flywheel, i.e. if the front end of the spring is in contact with the secondary mass, then $\sigma_{1}^{S}=1$ and if the back end of the spring touches the secondary mass stopper, then $\sigma_{6}^{S}=1$. The torque terms $M_{\text {fric,pri }}$ and $M_{\text {fric,sec }}$ describe all friction forces that act on the flywheels. Both 
friction terms are modelled in two effects: The first is occurs in bearings and sealings between flywheel parts. This friction torque is marked $M_{\text {seal }}$. And the second, more important, is the friction $M_{f r i c, i}$ which results from the spring's radial forces. This friction torques only act on $M_{\text {fric,pri }}[3]$.

$$
\begin{gathered}
M_{\text {fric, } p r i}=M_{\text {seal }}+\sum_{i=1}^{5} M_{\text {fric }, i} \\
M_{\text {fric }, \text { sec }}=-M_{\text {seal }}
\end{gathered}
$$

A homogeneous spring model is sufficient because it would not show a change in stiffness. Thus, the spring is divided into 6 elastic segments and five concentric lumped masses (Fig.4). The application of the law of conservation of momentum returns simulation equations $(i=1$, ..., 5) [4]:

$$
I_{i}\left(\varphi_{\text {pri }}+\varphi_{S, i}\right)=M_{S, i}-M_{S,(i+1)}-M_{\text {fric }, i}
$$

Fig. 4 Spring model with mass points and stiffness

We assume that the spring moments $M_{S, i} a M_{S,(i+1)}$ are linear. The spring moments can be calculated by defining the spring stiffness $k_{i}$ and the nominal spring length $\Phi_{n o m, 1}(i=1, \ldots, 6)$ as [4]:

$$
M_{S, i}=k_{i}\left(\Phi_{n o m, i}-\varphi_{S, i}+\varphi_{S,(i+1)}\right)
$$

The positions of springs $\varphi_{S, 0}$ a $\varphi_{S, 6}$, which have no mass points in the model, are based on the following equations:

$$
\begin{aligned}
& \varphi_{S, 0}=\max \left(\Phi_{P S}, \varphi_{D M F}-\pi+\Phi_{S S}, \varphi_{S, 1}-\Phi_{n o m, 1}\right) \\
& \varphi_{S, 6}=\min \left(\pi-\Phi_{P S}, \varphi_{D M F}-\Phi_{S S}, \varphi_{S, 5}+\Phi_{n o m, 6}\right)
\end{aligned}
$$

$\Phi_{P S}$ - half-width of the primary stopper

$\Phi_{S S}$ - half-width of the secondary 
When the side springs are not compressed, the elements $\varphi_{S, 0}$ a $\varphi_{S, 6}$ will render the outermost spring elements idle. Depending on the min/max functions in the equations (20) and (23), the switching can be calculated next section. To calculate the friction of the spring elements and the mass in the spring channel is necessary to figure out the radial forces $F_{R, i}$ which act on the mass elements. For each of the mass elements, different effects $i=(1, \ldots, 5)$ are taken into account [3].

$$
F_{R, i}=F_{\text {centrifugd }, i}+F_{\text {redirection }, i}+F_{\text {bias }, i}
$$

The centrifugal force at the effective radius of DMF is determined $i=(1, \ldots 5)$ :

$$
F_{\text {centrifugd }, i}=m_{i} r\left(\dot{\varphi}_{\text {pri }}+\dot{\varphi}_{S, i}\right)^{2} \approx m_{i} r \dot{\varphi}_{\text {pri }}{ }^{2}
$$

Redirection force for each of the spring elements $i=(1, \ldots, 6)$ is:

$$
F_{S, \text { defl }, i}=\frac{M_{S, i}}{r} 2 n_{w, i} \sin \left(\frac{\varphi_{F, i}-\varphi_{F,(i-1)}}{2 n_{w, i}}\right)
$$

$n_{w, i}$ - coils spring element

Redirection forces $F_{S, \text { def } l, i}$ are recombinant adjacent masses $i=(2, \ldots, 4)$ together with

$$
F_{\text {redirection }, i}=\frac{1}{2} F_{S, \text { defl }, i}+\frac{1}{2} F_{S, \text { defl },(i+1)}
$$

The redirection forces of the outermost springs, $F_{S, \text { defl,1 }}$ and $F_{S, \text { defl, } 6}$, are added to the forces which act at the outermost masses:

$$
\begin{aligned}
& F_{\text {redirection }, 1}=F_{S, \text { defl }, 1}+\frac{1}{2} F_{S, \text { defl }, 2} \\
& F_{\text {redirection }, 5}=\frac{1}{2} F_{S, \text { defl } l, 5}+F_{S, \text { defl }, 6}
\end{aligned}
$$

The bias term $F_{\text {bias, } i}$ models friction effects of an idle DMF [5].

$$
F_{\text {redirection }, 5}=\left\{\begin{array}{c}
F_{\text {bias }, 0}, i=1 \vee i=5 \\
0, \text { else }
\end{array}\right.
$$

Spring friction can be derived using the model:

$$
F_{\text {fric }, i}=\mu r\left|F_{R, i}\right|
$$

$\mu$ - friction coefficient

$$
F_{f r i c, i}=\left\{\begin{array}{c}
T_{a f f, i}, \quad \omega_{S, i}=0 \wedge\left|T_{a f f, i}\right| \leq \mu r\left|F_{R, i}\right| \\
\mu r\left|F_{R, i}\right| \operatorname{sgn}\left(T_{a f f, i}\right), \omega_{S, i}=0 \wedge\left|T_{a f f, i}\right| \leq \mu r\left|F_{R, i}\right| \\
\mu r\left|F_{R, i}\right| \operatorname{sgn}\left(\omega_{S, i}\right), \text { else }
\end{array}\right.
$$

Where $M_{a f f, i}$ is the difference of the two spring forces acting on the mass (20). It is clear from the equations that friction always acts against the direction of movement of each element of matter. To achieve complete cessation $\omega_{S, i}=0$ in numerical simulation, the Karnopp approach is used [3].

$$
\omega_{S, i}=\left\{\begin{array}{c}
0,\left|\omega_{S, i}\right|<\dot{\Phi}_{\min } \wedge\left|T_{a f f, i}\right| \leq \mu r\left|F_{R, i}\right| \\
\omega_{S, i}, \text { else }
\end{array}\right.
$$




\section{Multibody model of DMF}

The method of multibody systems utilizes a finite set of elements such as in our case rigid bodies, joints and bearings, dampers, and springs. The DMF CAD model was modelled in software Catia V5 (Fig. 6). Subsequently, the model was exported to the MSC Adams software environment in the form of STP files. The model consists of three main groups (the primary part of the flywheel, the second part of the flywheel and the spring caps and sliding shoes), which can be seen in Figure 5. Each component of DMF was assigned the required material density. This made it possible to determine the weight of the required part and thus calculate the inertial forces. The composition of all components created the required multibody model of DMF. Contacts between DMF parts were defined as geometric contacts between sliding shoes and spring caps, spring caps and ring gear (secondary part of the flywheel), spring caps and retainer platter (primary part of the flywheel).

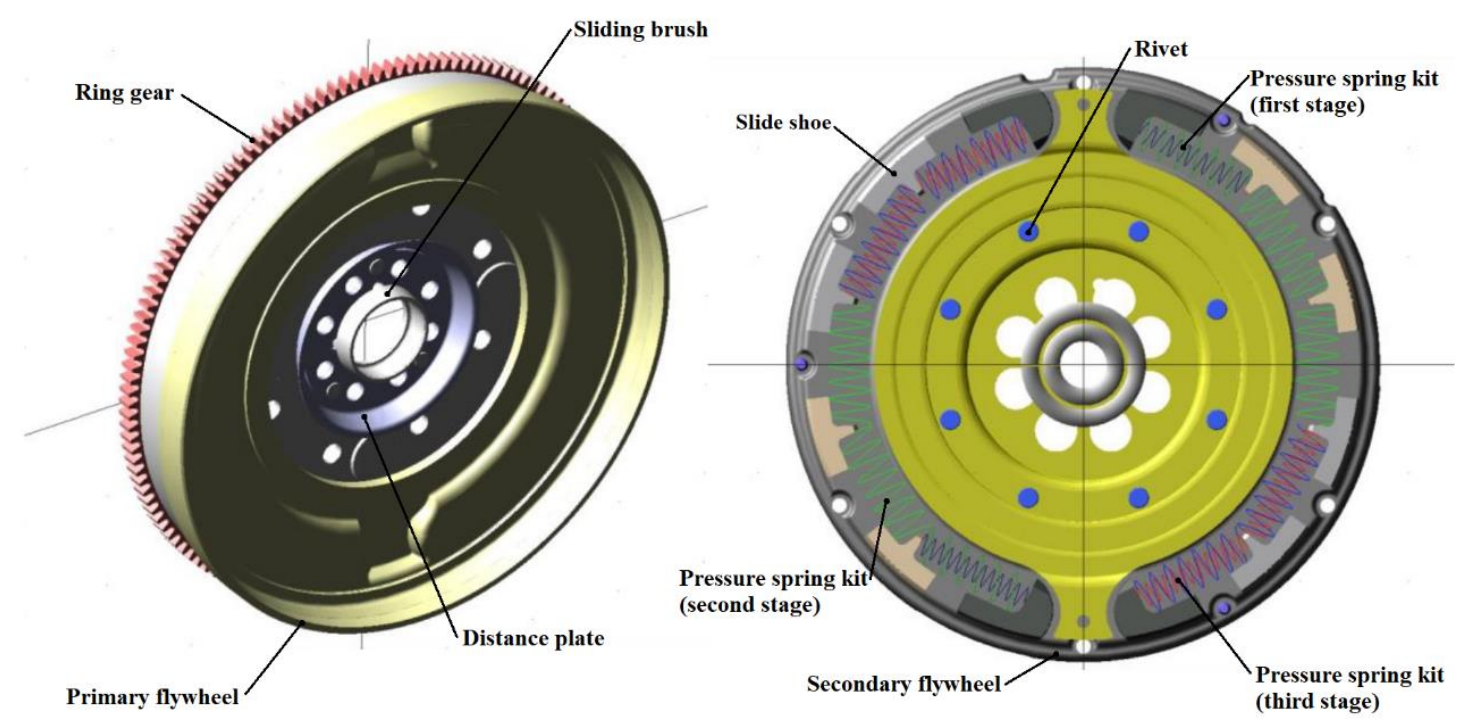

Fig. 5 DMF model in MSC Adams

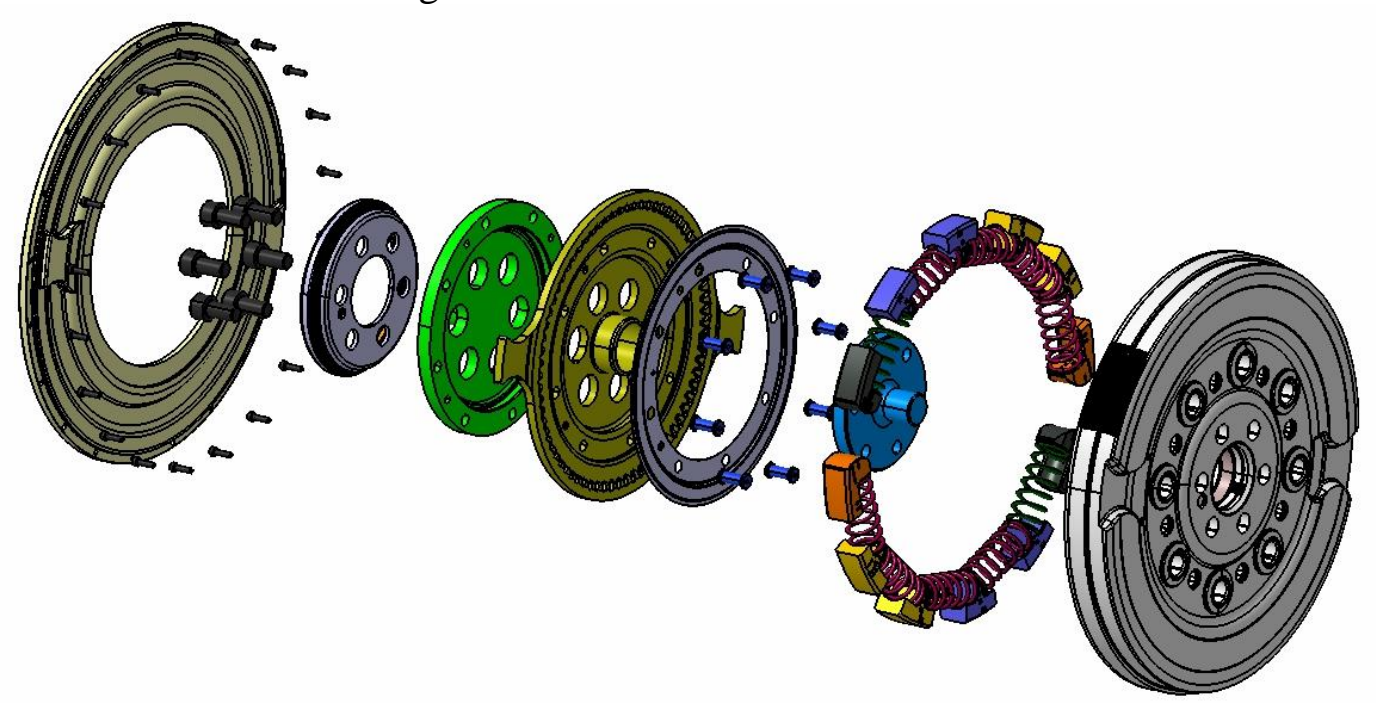

Fig. 6 CAD model in Catia V5

In DMF occurs lubrication grease in the spring space. It is used mainly to reduce friction, prevent wear, protect against corrosion but also absorbs shocks. In the simulation, it would generate hydrodynamic forces as nonlinear functions of the time parameters, while it would be 
necessary to evaluate the various dynamic parameters on which these forces depend. Due to experimental models in which the lubricant has been removed, it is not included in the simulation model either.

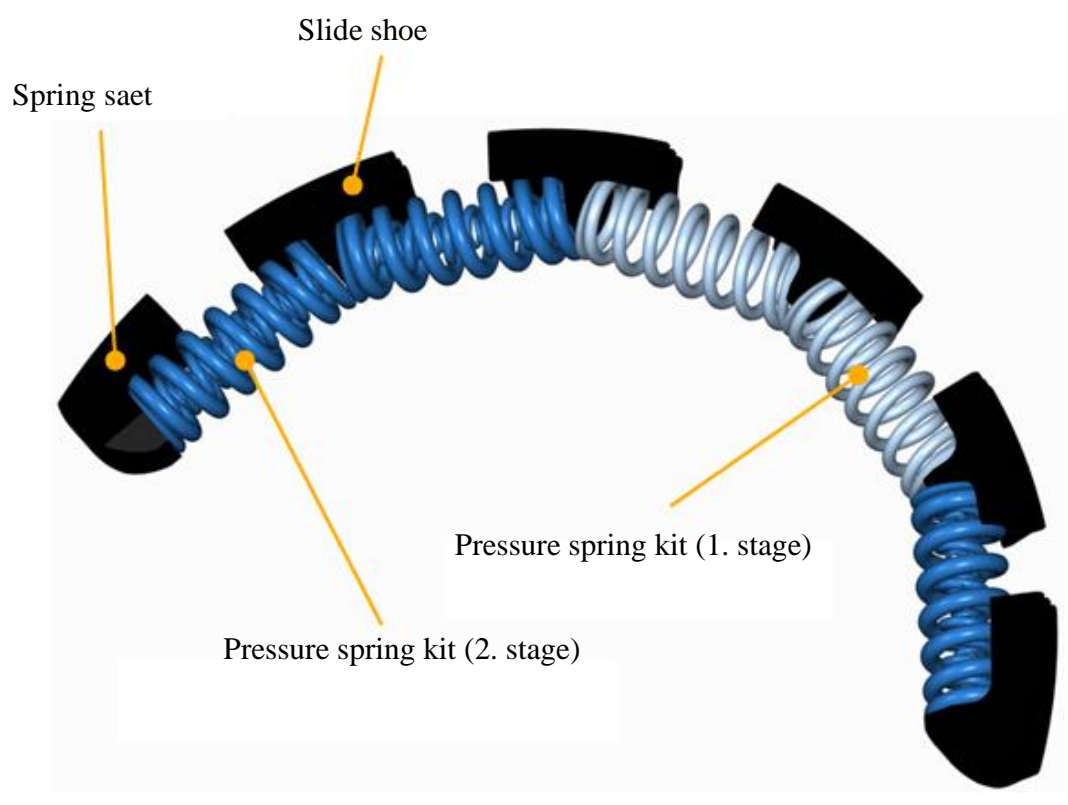

Fig. 7 Construction part of pressure spring set

\section{Experimental model system}

The measuring device can simulate the excitation of the torque generated by the motor and thus allow to measurement of the required parameters of the primary part and secondary parts of the flywheel is measured by second motor. The measuring test rig consists of two motors with position of rotation sensor. Torque moment is computed from electrical excitation of motor, so no native torque sensor is installed in system.

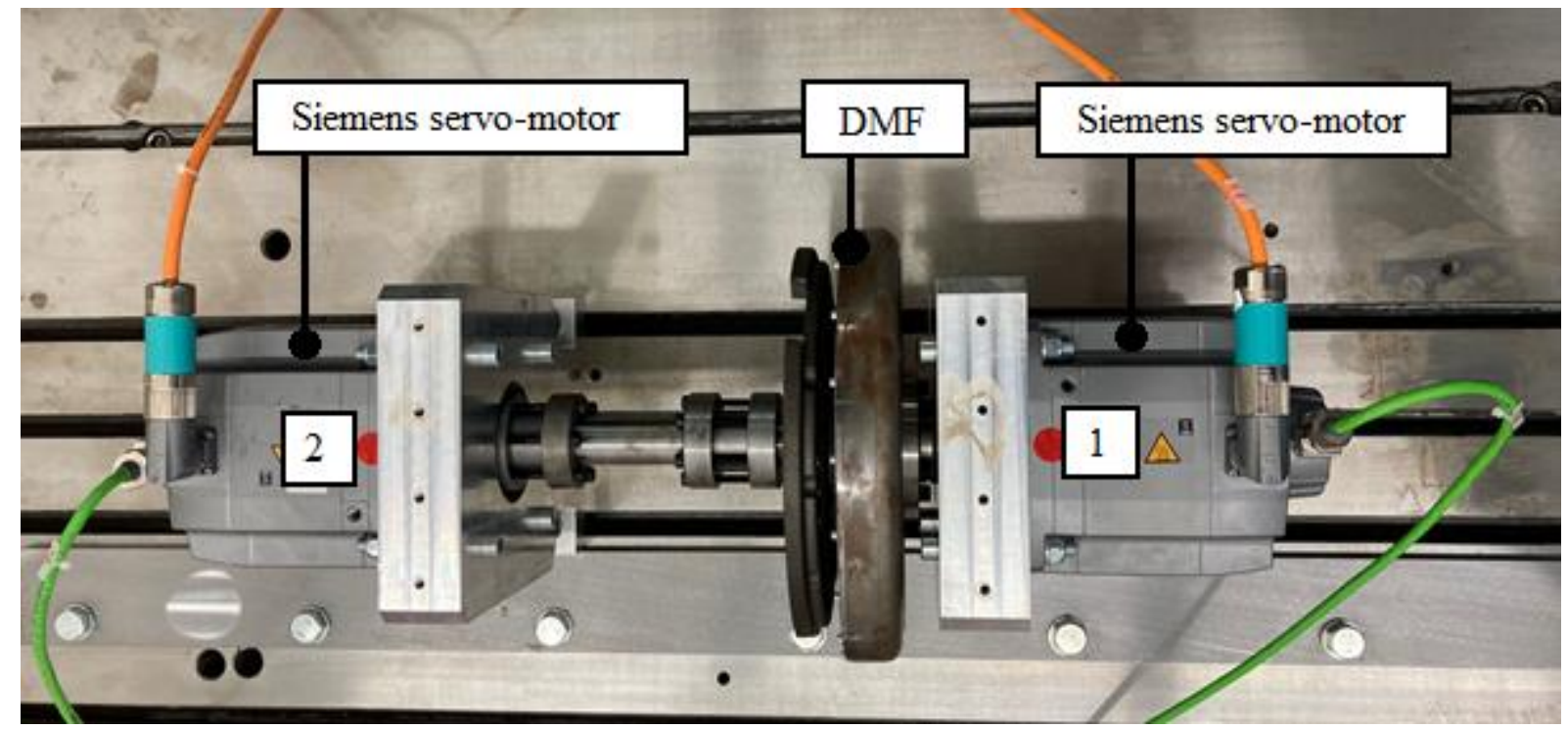

Fig. 8 Experimental test rig for verification of DMF model 
The main principle is based on measuring the differences in shaft angle between two motors. Test rig configuration is in Figure 10. The motor 1 is used as a mechanical torque source and generate real torsional excitation based on simulated torsional vibrations. The motor 2 is operated as a brake to create the required resistances. Energy from the motor 2 is recuperated and flow back to motor 1 via internal S120 recuperation mode. From the excitation servomotor, the power passes to the DMF and the subsequently transmitted power is transferred to the motor 2. The shafts of the experimental model are connected using a Chiaravalli coupling.

Selected motors are synchronous with permanent magnets. They have a rated power of 3.24 $\mathrm{kW}$ and a nominal torque of $13 \mathrm{Nm}$. They can be overloaded up to $40 \mathrm{Nm}$ in a short period of time, which meets the measurement requirements. An experimental device is controlled by system in combination with two real-time SIMATIC S7 system. It consists of two SIMATIC S7 1516 T controller and an integrated industrial PC SIMATIC S7. Due to wide compatibility, a second PC with TIA Portal V16 software was chosen, where Matlab / Simulink and PLC system is operated in real time.

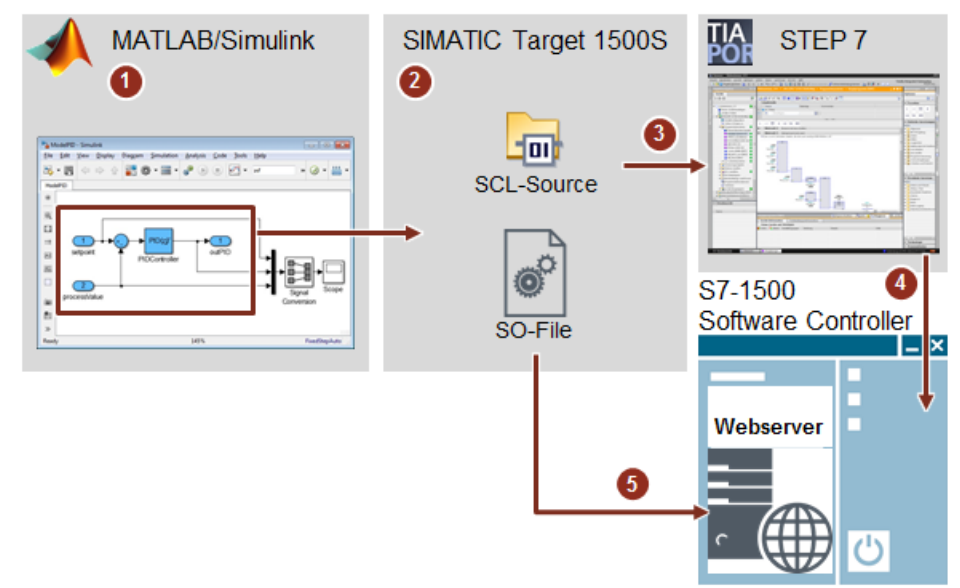

Fig. 9 System of Simulink - STEP 7 interface [6]

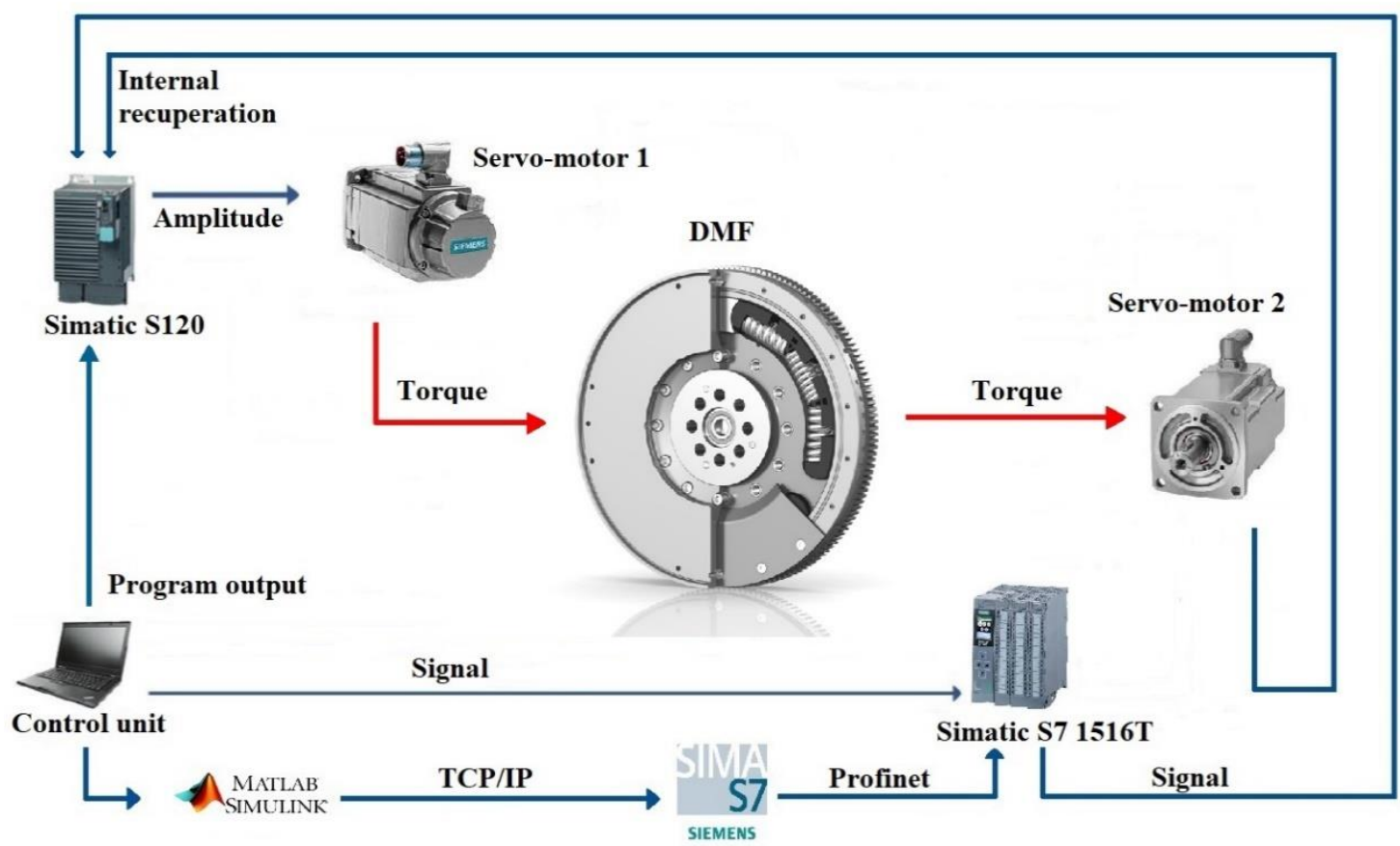

Fig. 10 Basic scheme of connection of parts and equipment 
The entire power control unit is stored in a rack cabinet. Complete scheme of realisation interface of all parts/units is in Figure 9. Sinamics S120 is controlled by Simatic S7 1516T unit. Industrial PC is connected to S7 1516T via Profinet with installed Simatic Target 1500S for Simulink and connection to $\mathrm{S} 120$ is done for reason of configuration.

\section{Comparison of virtual simulation with experimental DMF}

As mentioned above, the results are measured from the rotation differences between the two motors. In the first case, motor 1 produced a maximum angle of rotation of up to 13 degree in both directions and 26 degree in the entire range of rotation, respectively. Motor 2 generated excitation at the same speed with a delay of $0.05 \mathrm{~s}$ in the same direction. Measured angles of rotation differences are evaluated in Matlab via TCP/IP data transfer and logging (further only via graphical representation).

Parameter of virtual model has been done on theoretical basis (based on geometry) and should be tuned numerically to achieve acceptable match to reality - real measurement of experimental test rig. For this purpose, Simulink Design Optimization toolbox has been used. This procedure used MSC Adams co-simulation with Matlab in Simulink environment.

Because of multi parameters model of DMF, mainly friction parameters were selected to be tuned. For most exact simulations real data from experiments - from motor 1 - excitation was transformed to be input to simulation process in MSC Adams - Matlab/Simulink co-simulation. Then simulated results (from co-simulation MSC Adams - Matlab/Simulink) equivalent to secondary part motion (motor 2) were compared to measured data from motor 2.

On Figure 11 and 12 we can see results after tuning multibody model. On figure 11 is comparisons of rotation angle between Primary (Motor 1) and Secondary (Motor 2) parts of DMF - simulated and measured.

On Figure 12 is of Torque Moment on Primary (Motor 1) and Secondary (Motor 2) parts of $\mathrm{DMF}$ - simulated and measured.

On both figures we can see, that simulated values are lower to experimental measured. Also, there is small time delay in simulated values to experimental data. 


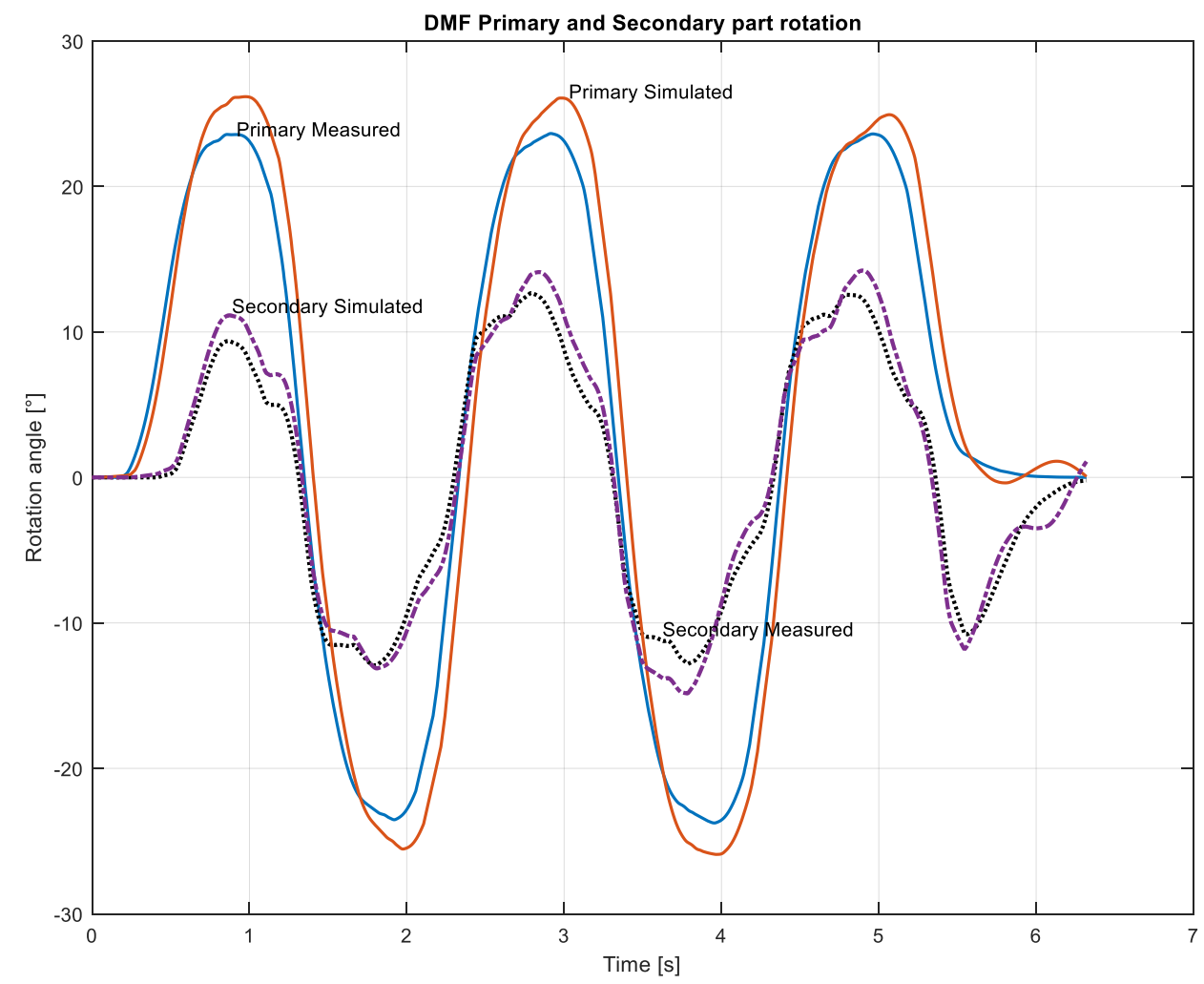

Fig. 11 Comparisons of rotation angle between Primary (Motor 1) and Secondary (Motor 2) parts of DMF - simulated and measured

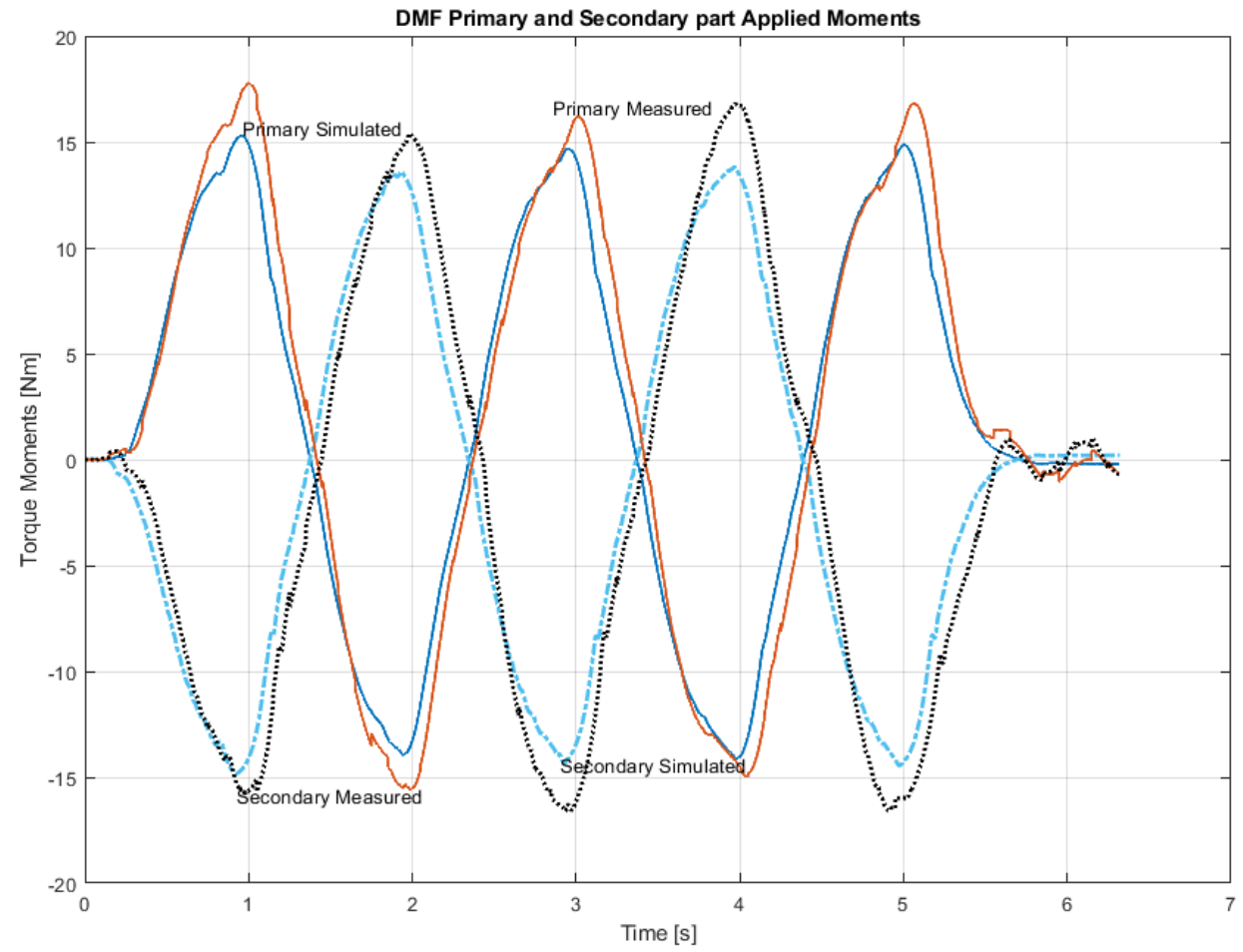

Fig. 12 Comparison of Torque Moment on Primary (Motor 1) and Secondary (Motor 2) parts of DMF simulated and measured 


\section{CONCLUSION}

From the compared results, (see Figure 10 and 11) we can see, that simulated model after process of tuning parameters is not $100 \%$ equal real model. Difference in statistical point of view is close to $91 \%$. For multibody model with 56 elements and 24 friction bindings it is very good result. Mainly friction bindings produce complicated solutions and analytical description of technical problem with numerical solution is unsoluble problem[7] in technical praxis. Multibody simulation process is only solution and nonlinear friction parameters tuning is difficult to realize to achieve acceptable results. Multibody modelling can reduce the cost and time of designing a dual-mass flywheel, which can be integrated into modelling of driveline system in automotive applications. It is not necessary to describe the process by mathematical equations, but as mentioned, the simulation in multibody environment is closer to CAD models. The concept can also be adapted to other sectors where torsional vibration occurs.

\section{ACKNOWLEDGEMENT}

This work was supported by the Slovak Research and Development Agency under Contract No. APVV-19-0406, and by Research Agency (Výskumná agentúra) ITMS+313011V334.

\section{REFERENCES}

[1] Quattromani, G., Palermo, A., Pulvirenti, F., Sabbioni, E., Cheli, F. "Nonlinear 3D Dynamic Model of an Automotive Dual Mass Flywheel“", In: Rotating Machinary, Hybrid Test Methods, Vibro-Acoustics and Laser Vibrometry 8, USA, pp. 131, 2017.

[2] Wedin, A. "Reduction of Vibrations in Egines using Centrifugal Pendulum Vibration Absorbers", Masters' Thesis, CHALMERS UNIVERSITZ OF TECHNOLOGY, pp. 7 11, 2011.

[3] Schaper, U., Sawodny, O., Mahl, T., Blessing, U. "Modeling and torque estimation of an automotive Dual Mass Flywheel”, American Control Conference, pp 6., 2009.

[4] Karnopp, D. "Computer simulation of stick-slip friction in mechanical dynamic systems", ASME DSMC, pp. 100 - 103, 1985.

[5] Albers, A. “Advanced Development of Dual Mass Flywheel (DMFW) Design”, LuK Symposium, pp. 1 - 38, 1994.

[6] "SIMATIC Target 1500S: Calling Simulink Models" (online), Available at: web https://support.industry.siemens.com/cs/document/109482830/simatic-target-1500scalling-simulink \%C2\%AE-models?dti=0\&lc=en-CN (12.09.2021)

[7] INMAN, D. J. "Vibration with Control", John Wiley \& Sons Ltd., 382 p., 2006. ISBN13 978-0-470-01051-8. 